\title{
Tuberculosis of the Greater Trochanter
}

\section{Report of Four Cases}

\author{
E. Mouhsine ${ }^{a} \quad$ S. Pelet $^{a} \quad$ M. Wettstein ${ }^{a} \quad$ C.-H. Blanc ${ }^{a} \quad$ R. Garofalo ${ }^{a}$ \\ N. Theumann ${ }^{b}$ O. Borens ${ }^{a}$
}

Departments of ${ }^{\mathrm{a}}$ Orthopedics and Traumatology and ${ }^{\mathrm{b}}$ Radiology, University Hospital, Lausanne, Switzerland

\author{
Key Words \\ Greater trochanter - Tuberculosis
}

\begin{abstract}
Objective: To present four cases of tuberculosis of the greater trochanter. Case Presentation and Intervention: The four cases ( 3 females and 1 male), aged 45-70 years, presented with mechanical pain in the trochanteric area associated with progressive swelling in the 3 female patients in whom mobility was also restricted. X-ray revealed a mass in 2 females; CT scan and MRI exhibited an abscess in the 3 females. Histological and bacteriological examinations showed Mycobacterium bovis in the 3 females and M. tuberculosis in the male. In the females, tritherapy and surgery were performed, while in the male quadritherapy and surgery. All the patients recovered and were followed up for 4-9 years. Conclusion: These cases show that both chemotherapy and surgery must be synergic if tuberculosis is diagnosed and an abscess is confirmed by imaging.
\end{abstract}

Copyright $\odot 2006$ S. Karger AG, Basel

\section{Introduction}

Tuberculosis remains widespread all over the world, though occurring mainly in underdeveloped countries, infecting over 10 million people. Two million people [1] were killed in 2001 and 3 million in 2002.
In Switzerland, 200 new cases of tuberculosis are detected every year. In more than $85 \%$ of the cases, the respiratory system is affected. Osteoarticular localization is not frequent (less than $1.5 \%$ of all cases of tuberculosis).

The main causative agents are Mycobacterium tuberculosis, M. bovis and M. africanum. Primary infection often goes undetected. Reactivation may occur several years or decades later and occurs as a consequence of a decrease in the patient's immunity [2]. This is sometimes linked to immunity-suppressive factors, i.e. disease, old age or drugs, such as chemotherapy agents for cancer treatment [3]. Secondary skeletal involvement mainly occurs at this stage. However, it is difficult to determine that period because it depends on immunity suppression. The osseous involvement is often the first manifestation of the disease and is not associated with reactivation of pulmonary tuberculosis. Of these cases, the greater trochanter is affected in only $2 \%$. This diagnosis is difficult to make, as clinical signs are nonspecific, radiographs are initially not useful [4], and patients presenting with a voluminous bone abscess are rare. Therefore, more invasive diagnostic techniques are required, and microbiological as well as histological examinations remain necessary to obtain a specific diagnosis. 


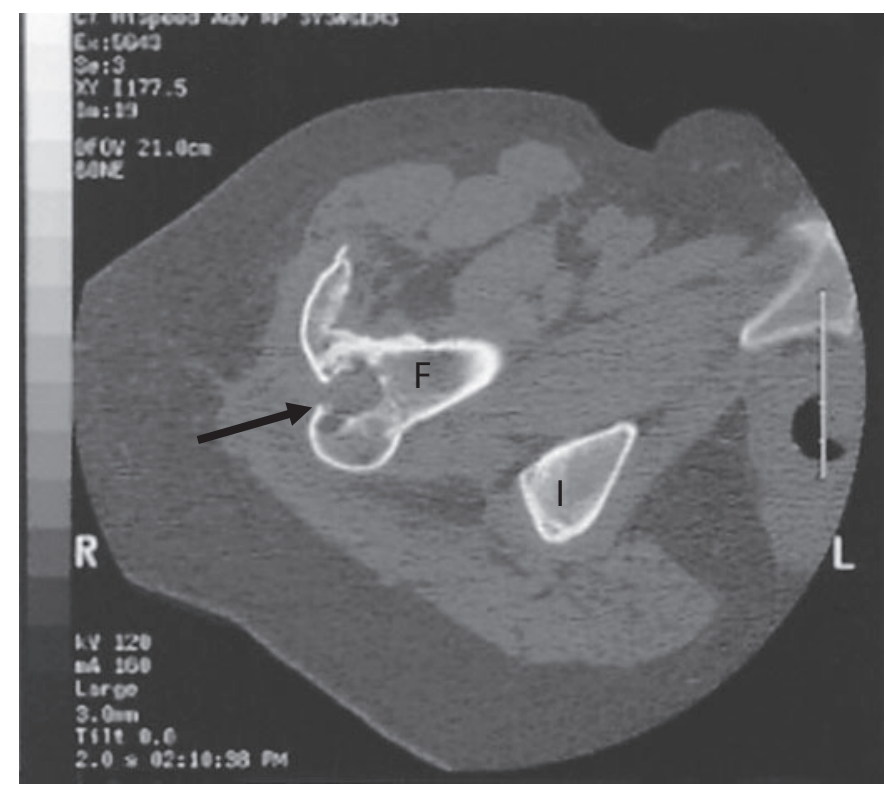

Fig. 1. Patient 1. Lytic lesion (arrow) of the greater trochanter on a CT scan. $\mathrm{F}=$ Femur; $\mathrm{I}=$ ischium.

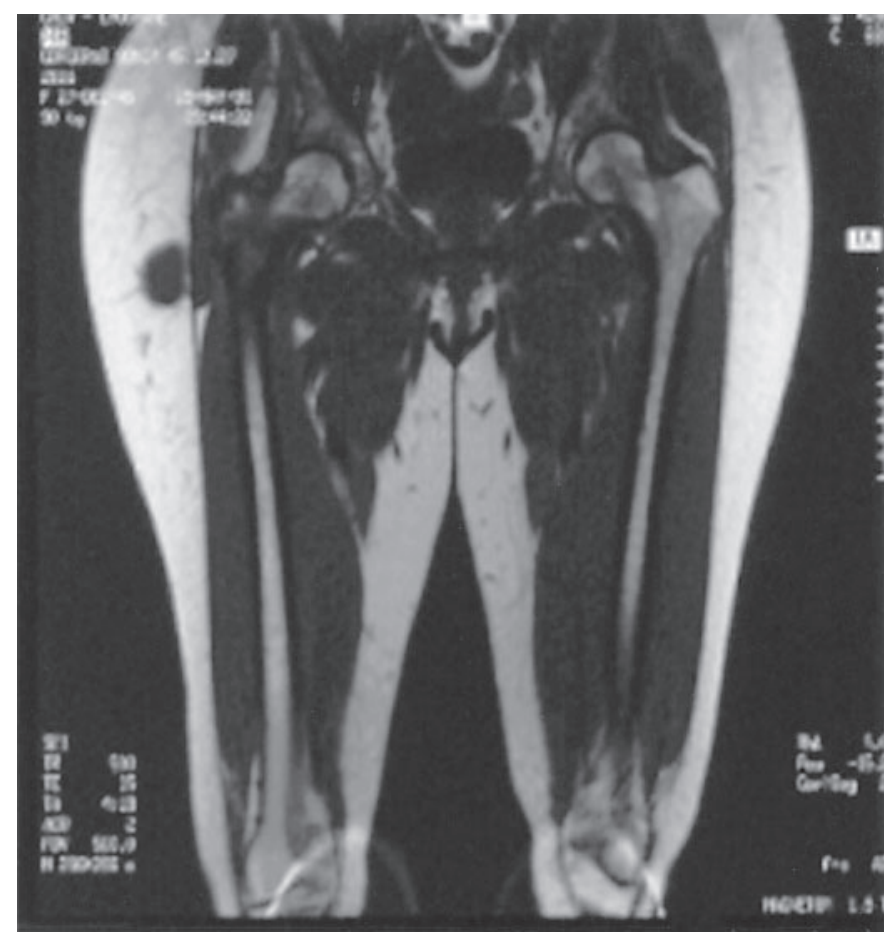

Fig. 2. Patient 1 . Bone lesion and subcutaneous abscess on MRI exam.

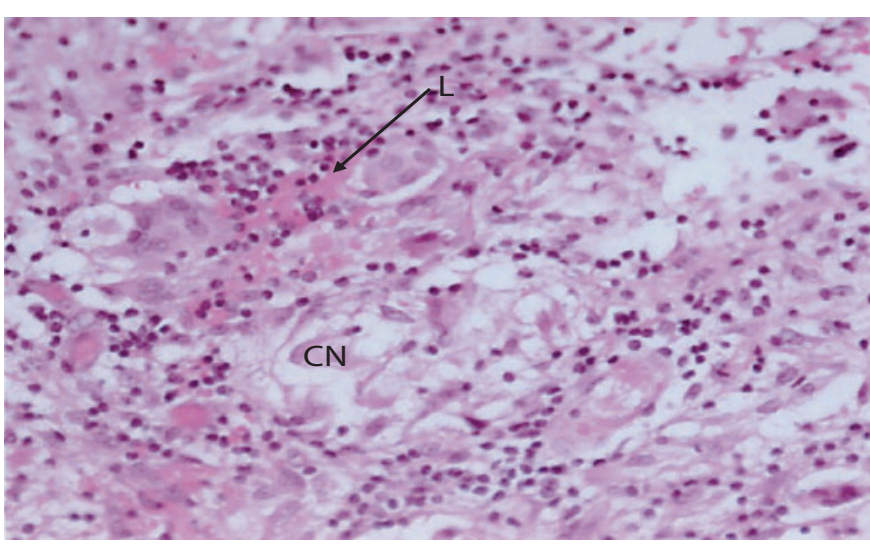

Fig. 3. Patient 1 . Histological sample showing a granuloma with caseous necrosis $(\mathrm{CN})$ and giant Langerhans cells $(\mathrm{L})$.

\section{Case Reports}

Case 1

A 45-year-old female patient presented with complains of mechanical pain in the right trochanteric area associated with progressive swelling of the lateral aspect of the thigh that made it impossible to lean on the right side. Hip mobility was unrestricted but a painful mass was palpated in the trochanteric region. The patient was in good general condition and neither had any notion of primary tuberculosis in her past nor any evidence of the disease in her medical history. Routine laboratory tests were within normal limits. Radiological images showed a modification in the structure of the greater trochanter with an irregular aspect of the lateral cortex and a soft tissue mass measuring $8 \mathrm{~cm}$ in diameter. CT scan showed a lytic process compatible with the abscess of the greater trochanter (fig. 1). MRI showed a communication between the subcutaneous abscess and the greater trochanter through the fascia lata (fig. 2). Needle aspiration of the abscess revealed $M$. bovis on cultures and a tuberculin test done at this time was positive. Antituberculous tritherapy (rifampicin, isoniazid, ethambutol) was introduced; sequestrectomy of the greater trochanter with evacuation of the subcutaneous abscess, excision of the trochanteric bursa and placement of gentamicin beads were done. Histological examination of the trochanteric bone revealed an inflammatory reaction with a granuloma with caseous necrosis and giant Langerhans cells confirming the diagnosis (fig. 3). No mycobacterium was detected. Chemotherapy was continued for 4 months, whereas the gentamicin beads were removed after 3 months. The clinical course was uneventful. No recurrence was observed after a follow-up of 6 years. 
Case 2

A 70-year-old patient presented with long-term mechanical pain in the left trochanteric region that was first diagnosed as untypical lumbosciatic pain because of associated degenerative spondylarthritis. Hip mobility was normal. There was no swelling or palpable tumor. He was otherwise in good general condition with normal laboratory findings. Standard X-rays showed a destruction of the greater trochanter. CT scan exhibited no abscess in the soft tissues. Sequestrectomy of the greater trochanter revealed a great amount of caseous material suggesting tuberculous osteitis. This diagnosis was confirmed by bacteriological samples (M. tuberculosis) and a histological exam. Quadritherapy was conducted for 2 months, followed by 4 months of bitherapy. However, osteitis recurred during the fifth postoperative month with a cutaneous fistula. Because of bacterial sensitivity in the first microbiological exam as well as for a local treatment, another surgical intervention was planned including a large sequestrectomy and placement of gentamicin beads. The operative samples remained sterile. Tritherapy was pursued for 6 months and no recurrence has been detected to this day after a 4-year follow-up.

Case 3

A 58-year-old patient was treated for hip pain lasting for 4 years, with five steroid infiltrations in the trochanteric bursa. Clinical examination, laboratory analysis and standard X-ray did not lead to a specific diagnosis. Following a CT scan examination, a sequestrectomy of the greater trochanter with histological and bacteriological examination confirmed the diagnosis of tuberculosis. Parallel to the tritherapy and after a minimal abscess aspiration was performed, a second surgical intervention was needed, and a final and large sequestrectomy was successfully done. Tritherapy was pursued for 6 months and no recurrence has been observed to this day after a 9-year follow-up.

Case 4

A 46-year-old female in good general condition presented with no evidence of primary tuberculosis, and a mechanical right trochanteric pain. A painful mass was palpated without any hip mobility restriction. Routine laboratory tests and radiological images were unspecific. A CT scan confirmed an abscess of the greater trochanter and MRI revealed a fistula. Needle aspiration of the abscess showed M. bovis on cultures and a tuberculin test done at this time was positive. Antituberculous tritherapy was introduced and a sequestrectomy, including the evacuation of the subcutaneous abscess, excision of the trochanteric bursa and placement of gentamicin beads, was performed. The clinical course was uneventful and no recurrence was observed after a 9-year follow-up.

\section{Discussion}

Tuberculosis is currently the leading cause of death due to infectious diseases worldwide and continues to increase in prevalence [5], particularly in underdeveloped countries. In developed countries, the most important predisposing factors for developing tuberculosis are infection with the human immunodeficiency virus $[2,6]$ and aging. Other predisposing factors include health care workers exposed to tuberculosis and the development of drug-resistant $M$. tuberculosis [5] due to a greater number of people traveling around the world and immigrating from developing countries [3]. This is especially true for elderly, chronically immunosuppressed patients as well as patients with acquired immunodeficiency syndrome.

Despite the introduction of systemic antituberculous therapy, the incidence of tuberculosis has increased substantially. However, because musculoskeletal tuberculosis is an uncommon manifestation, its diagnosis is usually not considered in the evaluation of patients with musculoskeletal symptoms, as tuberculosis most commonly affects the respiratory system. Extrapulmonary manifestations, such as in the urogenital or musculoskeletal systems, are involved in approximately $20 \%$ of all cases [5].

Musculoskeletal tuberculosis occurs in 1-3\% of all tuberculosis patients [3]. Tuberculous arthritis has been well described in the literature as commonly affecting the vertebrae and the large and medium-sized joints, particularly the hip and the knee [5].

Haefliger [7] reported that, in Switzerland, 200 new cases of tuberculosis are detected every year. In more than $85 \%$ of them, the respiratory system is affected and in less than $1.5 \%$ the osteoarticular system.

Tuberculous bursitis of the greater trochanter accounts for only approximately $1 \%$ of musculoskeletal tuberculosis [2], whereas hip tuberculosis represents approximately $15 \%$ of all cases of osteoarticular tuberculosis [8]. Therefore, based on statistical evaluation, the next case of greater trochanter tuberculosis in Switzerland could predictably be encountered in 16 years [9].

Diagnostic problems may arise and lead to a delay in treatment. Clinical practitioners should not omit tuberculosis from the multiple differential diagnoses of persistent bone and joint pain, such as tumor, bursitis, or inflammatory arthritis [4]. Osteoarticular tuberculosis should be suspected in the presence of chronic pain associated with swelling.

There are certain imaging features that help to differentiate tuberculous osteomyelitis from other bone and joint disorders with a similar presentation: the metaphyses of the long bones in the lower extremities, as well as the small bones of the hands and feet are most commonly affected. Most frequently, peripheral involvement of bones leads to cortical destruction and communicating juxtacortical abscesses, which can be seen on MRI. These are strong predictors of bone tuberculosis. Medullary involvement of bones is less common and may be of variable 
Fig. 4. Algorithm for the diagnosis and treatment of bone tuberculosis.

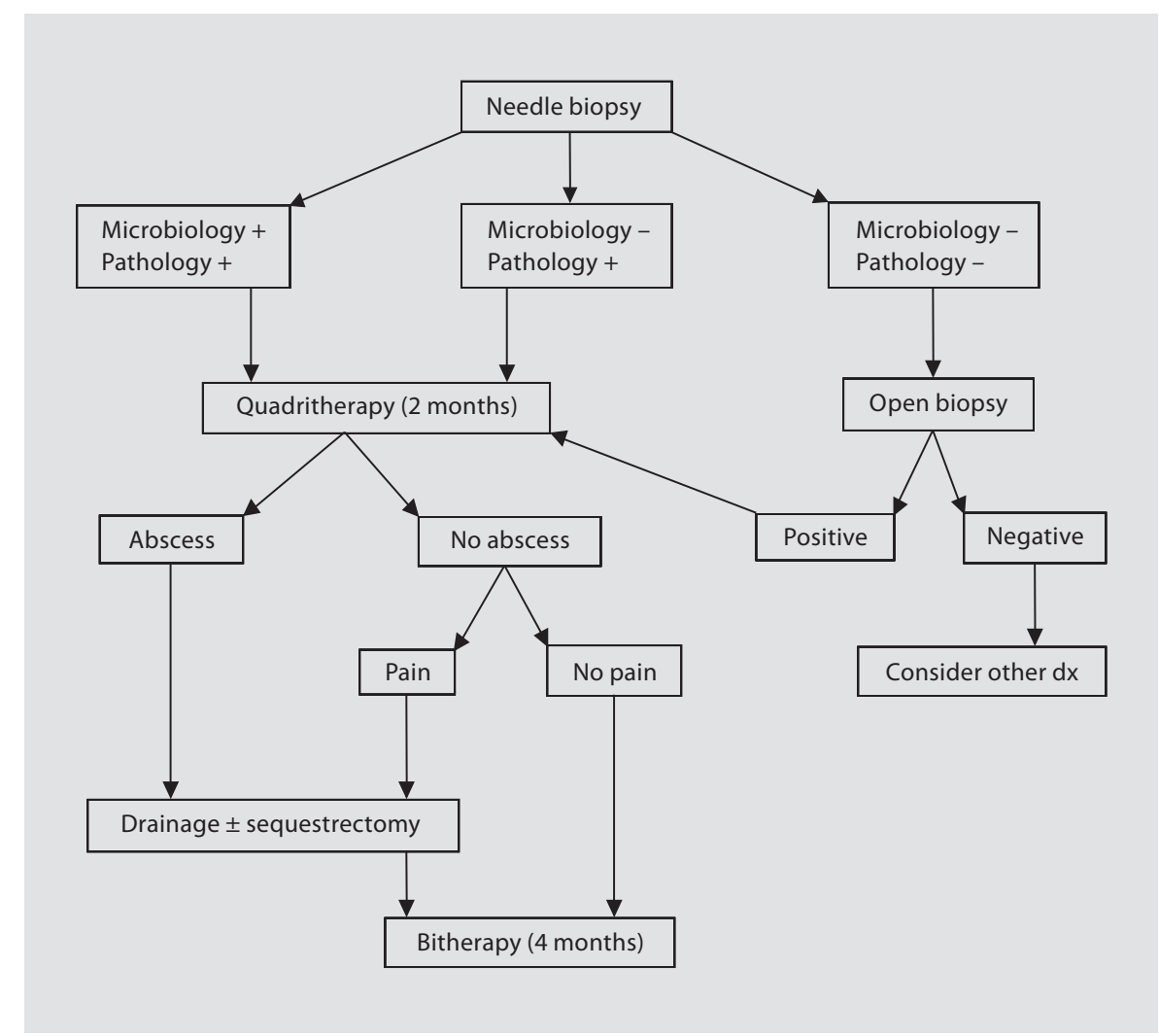

appearance. However, transepiphyseal spreading of the disease is more frequent in tuberculosis than other infectious diseases, thus being one distinguishing feature. The use of CT or MRI, coupled with aspiration or biopsy, should lead to earlier recognition of musculoskeletal tuberculosis, ideally before the onset of the debilitating disease $[4,9]$. An early diagnosis is of outmost importance, as approximately $90-95 \%$ of patients will achieve healing with near-normal function, if osteoarticular tuberculosis is diagnosed and treated at an early stage $[10,11]$.

A successful outcome depends on the use of the optimum regimen of chemotherapy, with safer and more effective drugs given regularly, and for an adequate period of time as in our four cases. The most frequent reason for failure is poor patient compliance with chemotherapy, thus leading to recurrence. The period of time necessary for adequate treatment seems to vary, and needs to be adjusted to each patient; variation ranges from 4 to 18 months in the literature $[10,11]$. We think that because of the complexity of this treatment and the use of new drugs, collaboration with infectious disease specialists is preferable. In our series, the use of an aggressive regimen with quadri- or tritherapy lasting between 4 and 6 months seemed adequate, as there was no recurrence after a follow-up of several years. However, as a recent article [12] showed, a recurrence may occur as late as 60 years after primary treatment. With actual treatment regimens combining chemotherapy and surgery, such a late recurrence seems less probable.

Surgical removal of infected tissue may be indicated to improve the efficacy of chemotherapy [10]. Surgery is also required if patients do not respond after 4-5 months of chemotherapy, if the therapeutic outcome is not satisfactory, or if treatment has resulted in a painful ankylosis [11].

Based on our experience with greater trochanter tuberculosis, and a review of the recent literature, we propose an algorithm for clinical evaluation of patients with suspected tuberculous bone disease (fig. 4).

\section{Conclusion}

Both chemotherapy and surgery must be synergic if tuberculosis is diagnosed and an abscess is confirmed by imaging. 


\section{References}

1 Ashford DA, Whitney E, Raghunathan P, Cosivi O: Epidemiology of selected mycobacteria that infects human and other animals. Rev Sci Tech 2001;1:325-337.

2 Yamamoto T, Iwasaki Y, Kurosaka M: Tuberculosis of the greater trochanteric bursa occurring 51 years after tuberculous nephritis. Clin Rheumatol 2002;21:397-400.

3 Ryuh-Sup K, Joung-Yoon L, Sae-Rom J, Kang-Yun L: Tuberculous subdeltoid bursitis with rice bodies. Yonsei Med J 2002;4:539542.

4 Babhulkar S, Pande S: Tuberculosis of the hip. Clin Orthop 2002;398:93-99. $\checkmark 5$ Ramanath VS, Damron TA, Ambrose JL, Rose FB: Tuberculosis of the hip as the presenting sign of HIV and simulating pigmented villonodular synovitis. Skeletal Radiol 2002;31:426-429.

6 Watts HG, Lifeso RM: Current concepts review: tuberculosis of bones and joints. J Bone Joint Surg Am 1996;78:288-298.

7 Haefliger E: Prevalence of tuberculous infection as an important indicator of endemic aspects and its continuous decline in the population - Past and future aspects. Schweiz Rundsch Med Prax 1999;45:18531865.
8 Babhulkar SS, Pande SK: Unusual manifestations of osteoarticular tuberculosis. Clin Orthop 2002;398:114-120.

9 Rachid K, Chkoura M, Moudene A: Localisation rare de la tuberculose osseuse. A propos de 3 cas. Rev Chir Orthop 2001;2:176179.

10 Shembekar A, Babhulkar S: Chemotherapy for osteoarticular tuberculosis. Clin Orthop 2002;398:20-26.

11 Tuli SM: General principles of osteoarticular tuberculosis. Clin Orthop 2002;398:11-19.

12 Sastre S, Garcia S, Soriano A: Reactivation of ancient trochanteric tuberculosis 60 years after surgical drainage. Rheumatology 2003; 42:1263-1264 